\title{
Study of theoretical models for the liquid-vapor and metal-nonmetal transitions of alkali fluids
}

\author{
E. Chacón ${ }^{1}$, J. P. Hernandez ${ }^{2}$, and P. Tarazona ${ }^{3}$ \\ ${ }^{1}$ Instituto de Ciencia de Materiales, Consejo Superior de Investigaciones Científicas and \\ Departamento de Física Fundamental, Universidad Nacional de Educación a Distancia, Apartado 60141, E-28028 Madrid, \\ Spain \\ ${ }^{2}$ Department of Physics and Astronomy, University of North Carolina, Chapel Hill NC 27599-3255 USA \\ ${ }^{3}$ Departamento de Física de la Materia Condensada (C-XII), Universidad Autónoma de Madrid, E-28049 Madrid, Spain
}

(May 3, 2019)

\begin{abstract}
Theoretical models for the liquid-vapor and metalnonmetal transitions of alkali fluids are investigated. Meanfield models are considered first but shown to be inadequate, apparently due to their inability to allow a microscopically consistent treatment of coexisting localization and delocalization of the valence electrons in the materials. An alternate approach is then studied in which each statistical configuration of the material is treated as inhomogeneous, with the energy of each ion being determined by its local environment. Nonadditive interactions, due to valence electron delocalization, are a crucial feature of the model. This alternate approach is implemented within a lattice-gas approximation which takes into account the observed mode of expansion in the materials of interest (a change in the average coordination rather than a change in the average nearest-neighbor distance) and which is able to treat the equilibrium density fluctuations. We have carried out grand canonical Monte Carlo simulations, for this model, which allow a unified, self-consistent, study of the structural, thermodynamic, and electronic properties of alkali fluids. Applications to $\mathrm{Cs}, \mathrm{Rb}, \mathrm{K}$, and $\mathrm{Na}$ yield results in good agreement with experimental observations.
\end{abstract}

PACS numbers: $61.25 . \mathrm{Mv}, 64.70 . \mathrm{Fx}, 71.30 .+\mathrm{h}$

\section{INTRODUCTION}

Until recently little has been known regarding the interrelation of the structural, thermodynamic, and electronic properties of metal-atom fluids. However, such knowledge has been being developed in a substantial body of experimental data which has been crying out for theoretical interpretation and guidance. The electronic, structural, and thermodynamic properties of such fluids have been shown to be intimately related and the interdependence of the metal-nonmetal and liquid-vapor transitions has posed a challenge to theoretical understanding. Detailed experimental studies are available for those materials with the lowest liquid-vapor critical temperatures: $\mathrm{Hg}(1751 \mathrm{~K})$, Cs $(1924 \mathrm{~K})$, and Rb (2017 K), with data on K (2178 K) becoming available most recentlye. The data have become precise and reliable in the last decade and span thermodynamic and electrical measurements under the same conditions. Such data show that the liquid-vapor coexistence curve of metal-atom fluids are different from those of Lennard-Jones-like ones] sumably due to many-body effects associated with valence electron delocalization. For example, the law of corresponding states is not obeyed when metal-atom fluids are compared with pair-interacting ones. Also, the liquid and vapor branches of the coexistence curves are strongly asymmetrical and the rectilinear diameter law breaks down over a substantial temperature range, not only very close to the critical points. These materials also undergo a metal-nonmetal (M-nM) transition. This body of data, however, still seeks microscopic theoretical foundationst. This paper presents a study of theoretical approaches seeking to comprehensively understand the alkali fluids. After demonstrating that a series of approaches, which would seem appropriate, fail to explain the general features observed, a simple model is described which does appear to contain the basic ideas required to reproduce, in a unified manner, the peculiar characteristics observed in the alkali fluids. This paper presents a detailed treatment of our results, improving and detailing the information in our recent letter $\mathrm{G}_{\text {. }}$

The goal of the present work - a unified understanding of the structural, thermodynamic, and electronic properties of metal-atom fluids - poses a considerable scientific challenge. Its various aspects are coupled since it is the electrons which determine interatomic interactions and thus the material structure and thermodynamic data. The ionic structure, in turn, determines the electronic properties. In the study of metal-atom fluids it is difficult to impose a structure, since it is so intimately related to electronic effects and there is no long-range symmetry to simplify the problem. Also, because in such materials the interactions are not pairwise ones for large and intermediate densities, due to electron delocalization over some regions, the problem is more complicated than that for simple, Lennard-Jones-like, fluids in which the interactions are not state dependent. Similarly, as these materials also undergo a M-nM transition, the traditional techniques used to study free-electron-like fluids are not applicable over many of the conditions of interest. The microscopic theory required for these materials should seek to explain the essential interdependence of thermodynamic, structural, and electronic properties.

Previous theoretical efforts to comprehensively explain the available experimental data on metal-atom fluids 
have been sparse. The points of view taken were usually based on the limiting cases of either a metallic, dense, liquid or solid, or alternatively of a nonmetallic, dilute, vapor. Attempts were then made to describe the fluid, or some of its properties, in a limited density and temperature range 6 . General arguments based on electron correlation effects (Hubbard model) and/or disorder induced localization (Anderson model) 9 are useful to study the M-nM transition in systems with frozen ionic structure but probably not in metal-atom fluids; at least, we are unaware of calculations attempting to link the structural, thermodynamic, and electronic properties using such methods.

A first step towards a theoretical treatment of metalatom fluids, which is intended to apply to both low and high densities at temperatures above approximately 2000 $\mathrm{K}$, extended concepts and techniques of plasma physics, in a mean-field approach, and introduced the required neutral atoms and small clusters 10 . These authors have recently claimed partial quantitative success in linking their calculations and a spectrum of experimental data. We also proposed such an approach 11 [12, not limited to high temperatures and including a discussion of the M$\mathrm{nM}$ transition. We showed that a toy model gives a liquid-vapor coexistence and a critical point with some correct features, but we were far from reproducing the peculiar coexistence curve of alkali fluids. In preliminary work 13 , we followed this approach including a quantitatively good description of the charged particle system: a standard description of liquid metals near their melting point 4 . Extension of this treatment to high temperature and low density gave a liquid-vapor coexistence with a very high critical temperature (around four times the experimental value), very low critical densities and pressures (by about an order of magnitude, compared to experiment), and a very different shape for the coexistence curve than that observed. To deal with the M-nM transition, we then extended the model, using a statistical treatment, to allow for chemical coexistence of neutral atoms with the ions. Phenomenological ion-atom interactions have been used, in work to be reported below, instead of the neglect of atom interactions assumed in the previous toy model. However, reasonable values of the parameters have not improved the previous results, in contrast to claims by others 10 . Details of this approach will be given in this paper. We have concluded that a mean-field theory is not capable of reproducing the structure or phase diagram of the alkali fluids. The physical reason for this failure is discussed below.

An unanswered question in a mean-field approach, with an atomic and a metallic component, is: Why do some fraction of the valence electrons choose to be bound in atoms while others are delocalized, at fixed temperature and chemical potential? The answer must lie in a hitherto ignored underlying structure. An important clue is that clustering effects are strongly enhanced for metalatom systems, compared to nonmetallic ones, due to their high cohesion, which arises from the valence electron de- localization over the cluster. In contrast, to retain its valence electron an atom should have no near neighbors to which that electron can be favorably delocalized. Further, experimental neutron scattering data15 have shown that the materials expand by changing their average coordination, rather than their average nearest-neighbor (nn) distance. This fact is especially important in systems in which valence electrons delocalize since such delocalization leads to contributions to cluster energies which go beyond merely additive effects in the local coordination; metal-atom clusters are strongly bound and their cohesion is a non-linear function of their density. Thus, in contrast to a mean-field characterization, structural and electronic effects are intimately related when equilibrium density fluctuations can be appreciable; this is the case in the expanded metal-atom liquid and vapor cases.

Based on the above, we have explored a model which takes into account, from the beginning, that expansion in the materials of interest takes place through a change in the average coordination, rather than by changing the $\mathrm{nn}$ distance, and which allows treatment of equilibrium density fluctuations. We have begun with the simplest model which seeks to give a recipe for the energy of an ion in a specific local environment, including effects due to possible valence electron delocalization. The recipe is to treat each local environment as a macroscopic one with an average density equal to that due to the ion in question and its local coordination. The possibility of valence electron localization, to form an atom, is taken into account by choosing the lower energy arising from treating the system as a metal of low density or an atom, dimer, etc. By treating each statistical configuration of the material as inhomogeneous, such a recipe provides the basis with which a self-consistent treatment of the material structure may be sought. To implement the self-consistent structural treatment, we have begun with a lattice-gas approximation and obtained its equilibrium properties using grand canonical Monte Carlo (MC) simulations. This approach yielded results showing the observed peculiarities of the alkali fluids 5 . That work has been improved and full details will be given here. Results for $\mathrm{Cs}, \mathrm{Rb}, \mathrm{K}$, and $\mathrm{Na}$ have been obtained and will be presented.

To orient the reader, we summarize the contents of this paper. In section II we discuss the mean-field treatment, for its own sake and with a view to an application discussed later. We begin discussing a hypothetical metal at an assumed arbitrary density. Thermal effects due to the delocalized electrons are examined. Pseudopotential parametrizations are discussed and compared. Hardsphere and one-component-plasma reference systems for the ions are compared and contrasted. Then, atoms are introduced in thermal equilibrium with the metallic component. Results, conclusions, and criticism of this approach follow. The crux of the model which proves successful follows. Section II discusses our approach and its implementation within the lattice-gas approximation. The grand canonical Monte Carlo treatment of the lat- 
tice gas follows; results of its application to the spectrum of alkali fluids are then given. Phase coexistence, structural features, and conductivity results are displayed and discussed. The paper concludes with a summarizing discussion and suggestions for further work.

\section{MEAN-FIELD TREATMENT}

\section{A. Metal}

At first, we consider a hypothetical system, entirely composed of ions and a neutralizing sea of valence electrons; this system exhibits a liquid-vapor transition. Atoms, with their localized valence electrons, in thermal equilibrium with the ions and delocalized electrons will be added as a next step. A mean-field treatment for a system of positive ions, at a mean density $\rho$, and delocalized, neutralizing glectrons is the normal one used for liquid alkali metals 14 . It is based on the Gibbs-Bogoliubov inequality:

$$
f \leq f_{o}+<H-H_{o}>_{o} / V=f_{o}+u(\rho),
$$

where $f$ is the free energy per unit volume $V, H$ is the system Hamiltonian, and the subscript indicates an ionic reference system. For the present problem we use an ionic reference system and a jellium treatment of the delocalized valence electrons, a pseudopotential $v_{p s}$ is then associated with the ions and the screened ion-electron and ion-ion interactions (minus reference system effects) are treated by perturbation theory. Hence, we can write:

$$
\begin{array}{r}
u(\rho)=u_{s i}\left(\rho_{e}\right)+ \\
2 \pi \rho^{2} \int_{0}^{\infty} d r r^{2} g_{0}(r ; \rho) \phi\left(r ; \rho_{e}\right),
\end{array}
$$

where $\rho_{e}=Z \rho$ is the electronic density, $u_{s i}$ is the sum of all structure-independent terms including the kinetic, exchange and correlation electronic energies of the jellium reference system and the first order pseudopotential perturbation term; $\phi\left(r ; \rho_{e}\right)$ is the total interatomic potential which is given by:

$$
\begin{array}{r}
\phi\left(\left|\mathbf{R}-\mathbf{R}^{\prime}\right| ; \rho_{e}\right)=\frac{Z e^{2}}{\left|\mathbf{R}-\mathbf{R}^{\prime}\right|}+ \\
\int d \mathbf{r} d \mathbf{r}^{\prime} v_{p s}(|\mathbf{r}-\mathbf{R}|) \chi\left(\left|\mathbf{r}-\mathbf{r}^{\prime}\right| ; \rho_{e}\right) v_{p s}\left(\left|\mathbf{r}^{\prime}-\mathbf{R}^{\prime}\right|\right),
\end{array}
$$

where $\chi$ is the electronic linear response function. For the ions, a hard-sphere (HS), or alternatively a onecomponent-plasma (OCP), reference gives entropy contributions $f_{o}$ and a pair distribution function $g_{o}(r ; \rho)$. If using the HS reference for the ions, one makes use of the Gibbs-Bogoliubov inequality by choosing the ion HS radius to minimize the system free energy at the density and temperature which correspond to the melting point for the liquid metal. This radius can also be used to minimize the free energy at higher temperatures, though the change in the obtained radius is negligible except at high densities. In the OCP choice, the ionic system is described via the parameter $\Gamma=(4 \pi \rho / 3)^{1 / 3} e^{2} / k_{B} T$, which is not treated as a variational parameter. The free energy estimates then allow discussion of the liquid-vapor phase diagram of the hypothetical material. Model results can be judged in a partial manner, here and later, by their approximate predictions for the vapor-liquid critical parameters; when such parameters are reasonable, a more detailed comparison with experiment can be sought.

In our calculations, thermal effects on the delocalized electrons are not taken into account. However, these effects had been probed in calculations of the phase diagram of a delocalized electron system, neutralized by jellium, in the temperature-dependent Hartree-Fock approximation 16; an ideal-gas reference for the ions was also incorporated. Such a treatment yields a critical point at $2550 \mathrm{~K}$ with a density of $1.3 \times 10^{-4}$ (a.u.). Replacing the temperature-dependent electron gas with a zero-temperature one, and maintaining exchange as the only net interaction, resulted in an increase of about $100 \mathrm{~K}$ in the critical temperature, a near-negligible correction. Further, if the correlation energy due to the zero-temperature electron gas is incorporated into such a calculation, the critical point temperature rises substantially. An ion ideal gas added to a zero-temperature electron gas, which includes correlations, gives critical parameters of $4184 \mathrm{~K}$ and $1.8 \times 10^{-4}$. Use of Padé approximants 10 , for the finite temperature electron gas, only changes the previous coexistence curve slightly, with the critical conditions then being given by $4500 \mathrm{~K}$ and $1.6 \times 10^{-4}$. Such, ten per cent, corrections suggest that thermal effects due to the electron gas are not important to the calculation of near-critical conditions in these systems.

Pseudopotential effects are then required. In the study of alkali liquid metals near their melting point, it has been common to simplify the ionic pseudopotential, which characterizes the material, by choosing one which is bcal and energy independent. In our previous calculations 5 we used the empty-core Ashcroft pseudopotential. Here we investigate the differences which arise from that choice and a Shaw pseudopotential (constant in the core and continuous at the cutoff radius). Both pseudopotentials only depend on one parameter, which is chosen to fit some experimental result for the liquid at the melting point, and then checked against a set of other experimental features. The set of experimental data of interest at the melting point consists of: the packing fraction (since the hard-sphere diameters are fixed to minimize the energy at the experimental density), the ionization potential of the atom (as a pseudopotential should reproduce the energy spectrum of states outside the core), the cohesive energy, the electrical conductivity (using the Ziman formula), the excess entropy over that of the ideal gas, and the pressure (one atmosphere). A common treatment of liquid metals 14 uses the Ashcroft pseudopo- 
tential fitted to the conductivity; many of the parameter choices reflect a fit to the conductivity in the solid. However, for some liquid alkali metals, such parameter choices yield a nonphysical packing fraction and are then reparametrized to fit a packing fraction of 0.45 . This was the parametrization we used in previous work 5 . However, in attempting to deal with the alkali family, rather than only one or two alkalis, we decided that fitting the presure at the melting point should be preferable. Also, we noticed that the previous parametrizations tended to substantially overestimate the cohesive energy of the liquids (relative to separated atoms) at the melting point. After examining the results of such parametrizations, and requiring that adjustments of the parameter to fit one property not result in spoiling the agreement with others, we have opted to use the alternate Shaw approach, fitted to the experimental pressure at the melting point. The ionization potentials, the cohesive energies, and the pressure are improved and we believe that such improvements are important for our purposes. Table I compares the results of the Ashcroft pseudopotential (fitted to the packing fraction or conductivity) and the Shaw alternative (fitted to the pressure) when applied to the liquid alkali metals at the melting point. We shall later compare results we have obtained using both approaches in the present application.

We have also explored the relative effects of the HS and OCP reference systems for the ions. There are two features which have an influence on the results obtainable: the excess entropy beyond the ideal gas, and the pair distribution function which enters in calculating the screened pseudopotential contributions. We have found that the excess entropy term is substantially different in the two choices of an ionic reference system, while the pair distribution and associated interaction energies are not a strong cause of differences between them. One must be careful in the comparison to note that, if the screened pseudopotential terms are excluded, the HS system only has the excess entropy but the OCP has such a term and the classical interaction of the ions in the electron sea. To quantify the effects of the ionic reference system, we have performed a series of calculations. We first sought the temperature and density of the critical point of an unspecified metallic system, excluding the screened pseudopotential terms, with the electron system treated at zero temperature. The HS reference yielded: $4200 \mathrm{~K}$ and a density of $1.8 \times 10^{-4}$; it should be noted that these results are almost identical to those using an ideal-gas reference, thus the excess entropy of HS over the ideal gas has a very small effect on the critical parameters, the density being so small. The OCP, however, yielded: 10600 $\mathrm{K}$ and $3.2 \times 10^{-3}$; the strong effect of the ionic interactions in the OCP leads to the higher critical temperature. Then, the effects of the screened pseudopotential were introduced. The results are then as follows, if one uses parametrizations with an empty-core and a cut-off radius of 1.70 a.u. (appropriate for Na). For HS, the calculated critical parameters change to $9000 \mathrm{~K}$ and $6 \times 10^{-5}$. For the OCP, the results become $4600 \mathrm{~K}$ and $1.5 \times 10^{-4}$. The interactions raise the HS critical temperature, while the partial cancellation of the classical ion interactions lowers that appropriate to the OCP. Since, recalling the GibbsBogoliubov inequality, the free energy estimates are an upper bound to the correct ones, obviously the better reference system is that which yields a lower value for the free energy. Hence, it is of interest to compare the free energies themselves, instead of focusing on a comparison of the above results to experimental data. Fig. 1 shows the free energy difference, with the sodium parametrization as an example of the result typical of all alkalis, for HS minus OCP as a function of density for three temperatures. It is clear that for the density range of interest here, $\rho>10^{-5}$, the HS reference system is to be preferred, having a substantially lower free energy of one to three $\mathrm{gV}$ per particle. A criticism of previously noted work 10 is that it uses the OCP reference system.

In table II we present the critical parameters, for the hypothetical alkali metals (labeled as MF-hs), obtained with the Shaw parametrization, the HS reference system, and the electronic system treated at zero temperature. In fig. 2 we show the coexistence curve for rubidium, obtained with the Shaw and the Ashcroft parametrizations; the difference here is relatively small. Also, it is clear that this type of result is in poor agreement, quantitatively and qualitatively, with experimental observations 13 . These results are typical of all alkalis. However, there are no atoms in the system, an important feature according to previous work 10,11 and crucial to incorporate the M-nM transition observed.

Since, physically, at moderately low densities the system must have the valence electrons localized on the ions but delocalized at high densities, the valence electrons must coexist in localized and delocalized states at fixed total chemical potential, pressure, and temperature, for phase coexistence. Thus, we proceed to consider a thermodynamic equilibrium mixture. We will restrict the mixture to a metallic component, as above, and atoms, without further complicating the treatment by considering diatomic molecules or other types of aggregates in this mean-field approach.

\section{B. Equilibrium with atoms}

The general approach followed here is along the lines previously noted 11 . The procedure uses an approximation to the system free energy in which ideal-gas terms for ions and atoms are first explicitly separated, the remainder of the free energy is denoted as $f_{e}$ :

$$
\begin{array}{r}
f \equiv k_{B} T\left[\rho_{a}\left(\ln \left(\rho_{a} \Lambda^{3} / 2\right)-1\right)+\rho_{i}\left(\ln \left(\rho_{i} \Lambda^{3}\right)-1\right)\right]+ \\
f_{e}\left(\rho_{i}, \rho_{a}, T\right) .
\end{array}
$$

The atom and ion densities are denoted by $\rho$, with appropriate subscripts, and $\Lambda$ is their thermal de Broglie 
wavelength. Then, the grand potential per unit volume is given by:

$$
\begin{array}{r}
\Omega / V=f-\mu_{a} \rho_{a}-\mu_{i} \rho_{i}= \\
f-\frac{E}{2}\left(\rho_{a}-\rho_{i}\right)-\frac{\mu_{a} \rho_{a}-\mu_{i} \rho_{i}}{2}\left(\rho_{a}+\rho_{i}\right),
\end{array}
$$

where $\mu$ are the chemical potentials and $\mathrm{E}$ is the vacuum ionization potential of the atom in question. $\Omega$ is first extremized with respect to the difference between atom and ion densities, with the sum of the densities being kept constant. The result of this procedure is an equilibrium relation between atom and ion densities:

$$
\rho_{a}=2 \rho_{i} \exp \left[\left(\mu_{e}+E\right) / k_{B} T\right],
$$

where $\mu_{e}=\partial f_{e} / \partial \rho_{i}-\partial f_{e} / \partial \rho_{a}$. Using this equilibrium relation then leaves the sum of atom and ion densities and the temperature as the only variables which can yield a liquid-vapor transition of the composite system. A jump in the relative ion to atom density, at fixed sum of densities, characterizes a resulting, first-order, M-nM transition, but this transition, and its critical point, can only take place hidden under the liquid-vapor phase coexistence of the composite system.

At first we considered a free energy with contributions from the electron gas, a neutralizing ionic system, and an ideal gas of atoms, with the atoms experiencing no interactions with the charged-particle system; thermal equilibrium with the metallic component was demanded. The work previously reported 11 showed that such an introduction of atoms would merely shift the critical point to higher density and pressure, from the conditions obtained without the atoms, without affecting the critical temperature. Although that work included only the electronic kinetic energy and exchange in $f_{e}$, the addition of correlation energies and the ionic terms would leave the above conclusion unchanged: the introduction of an ideal gas of atoms in thermal equilibrium with the charged particle system yields a liquid-vapor critical temperature which is that due to the system of charges and is independent of the ionization potential of the atoms. It has already been noted that such a temperature is extremely high, compared to the experimental results.

We proceeded, in a modification appropriate to Cs, by merely adding a HS reference for atoms with a diameter (10 a.u.) different than that of the ions (previously noted to be 8.80 a.u.); we used the method of Mansoori et al17. The dashed line of fig. 3 shows the coexistence curve obtained. A very high critical temperature resulted, and moreover the shape of the coexistence curve became clearly unphysical: with a density for the vapor branch nearly independent of the temperature and pinned by the mean-field, first-order, M-nM transition line. We conclude that only atom-atom and atom-charge interactions can change the critical temperature appreciably from that due to the charged particle system. The effect of such terms is to influence the relative populations of the atomic and metallic components in the system, as thermodynamic equilibrium between them is demanded.

As the interactions due to atoms tended to affect the coupling between the M-nM and liquid-vapor transitions, our main purpose in this investigation was to observe the influence of the atom interactions on the liquid-vapor critical point of the system. To carry out this investigation in full detail is quite complicated; for example, to include the polarization of atoms by the charged particles requires calculation of a micro-electric field distribution. The polarization energy of a neutral atom arises from the square of the sum of the (vector) electric fields to which it is exposed; it cannot be written as the sum of interaction energies with the individual charged particles (in contrast to previous assumption 10 ). Given this complication and also the fact that a pseudopotential treatment of atomelectron interactions has doubtful validity (as it treats the localized valence electron as frozen), we decided to begin by investigating a phenomenological treatment for the atom-charges interactions: a sum of a constant coefficient times the square of the atom density plus a second constant times the product of the atom density and a power of the charged particle density; the second term would be of dominant importance. Various exponents in the second term were considered.

Treating the atoms as hard spheres, with a diameter different than that of the ions, and further including (in $f_{e}$ ) their virial interaction with the charges, $a \rho_{a} \rho_{i}$, gives a first example of the type of effects obtainable. We used the empirical prefactor $a$ as a free parameter and analyzed the changes it induced in the critical temperature. This temperature, as a function of $a$, has a minimum which is less than five percent lower than the value with $a=0$, for whatever value may be chosen for the HS diameter of the atoms. Thus, this virial type effective interaction between ions and atoms can easily increase the critical temperature, but it cannot lower it in any substantial amount. We next studied the effects of other empirical forms for the interactions; for example $a \rho_{a} \rho_{i}^{\beta}$ with $\beta=2 / 3$ and $1 / 3$, which could conceivably result from the interaction between atoms and delocalized electrons. Again using $a$ as a variational parameter to minimize the critical temperature, we found that the critical temperature may be lowered by a significant amount (up to 60 percent) for $\beta=1 / 3$ and $a$ around -1900 a.u.. The full line of fig. 3 shows the coexistence curve for a cesium parametrization of the metallic component and this empirical form for the intactions with the atoms, with the optimal value of $a$ being used. Although the shape of the coexistence curve clearly improves compared to including the atoms only as hard spheres, the curve is far from having the experimental shape. Moreover, the critical temperature is still too high, even with the parameter $a$ taking the above, unphysical, value. The optimal value for $a$ implies that, at the critical density, an atom in the system has an energy which is $2.5 \mathrm{eV}$ below that of a free atom; in contrast, an electron ion-pair are bound to the metal at the melting point (relative to a 
free atom) by less than one $\mathrm{eV}$. We conclude that such, atom-charges, effective interactions are unable to bring the mean-field results into quantitative agreement with experimental observations.

\section{Criticism}

The above description of our efforts to integrate the M-nM transition with that of the liquid-vapor one suffers from internal inconsistencies as well. Not only did our efforts fail to yield results in semi-quantitative agreement with observations, but also it is hard to understand how a mean-field treatment can adequately describe the coexistence of localized and delocalized occupation of the states available to the valence electrons in the systems of interest. Clearly the statistical mechanics of the treatment are a straight-forward demand for thermal equilibrium between valence electrons localized in atoms and coexisting delocalized valence electrons, but there is a lack of a microscopic description which can allow for this coexistence. It seems clear that density fluctuations are the microscopic element which permits such a coexistence. Then, structural features can allow for valence electron localization in atomic states or a sharing of valence electrons, which will lead to metallic properties when the delocalization becomes macroscopic. Occupation of the atomic valence state requires a structural environment unfavorable to delocalization, that is a low density local environment. In contrast, high density local environments are favorable to valence electron delocalization on energetic grounds. On this basis, and recalling that the data relevant to the expansion of liquid metals (a change in coordination) is in conflict with a mean-field approach, we decided to investigate a model which explicitly considers equilibrium density fluctuations and their implications. The crucial point, however, is to calculate the energy of the system not in mean field but taking into account the strong inhomogeneities due to clustering and atom formation. We believe that the energy of each ion can be represented as depending on the local density of its environment; the functional dependence of this energy is extremely important in determining the resulting material structure and phase coexistence.

\section{MODEL}

\section{A. Configurational energies}

As previously noted, our main problem consists in being able to calculate the energy of a given statistical configuration of the system. Such a configuration will be inhomogeneous, containing regions of high local density (clusters), in which the valence electrons are delocalized over the region, and regions with the valence electrons localized on the ions, i.e. atoms. Clearly, on trying to calculate the energy of such a configuration, one may not use pair interactions, as the energy in the clusters is far from being describable in this manner. Similarly, using a macroscopic mean-field average, with the energy as function of the macroscopic average density, has already been discussed and found unsatisfactory. Thus, we proceed to take into account the inhomogeneity of the configurations in the following way. We describe the energy of a configuration by the sum: $U_{c}=\Sigma u_{i}$, over all the ions in the system $i=1, . . N$, and look for a workable approximation for the contribution of each ion, $u_{i}$.

The mean-field approach, described in the previous section, takes the energy $u_{i}$ to be a function of the global density $\rho$ and is thus equal for all the ions in the configuration, $u_{i}=u(\rho)$. On the other hand, in a system with pair interactions, the exact form for $u_{i}$ is given by the integral of the pair potential, $\phi(r)$, with the local density around the ion:

$$
u_{i}=\frac{1}{2} \int d \mathbf{r} \phi\left(\left|\mathbf{r}-\mathbf{r}_{\mathbf{i}}\right|\right) g\left(\mathbf{r}, \mathbf{r}_{\mathbf{i}}\right) \rho(\mathbf{r}),
$$

where $g\left(\mathbf{r}, \mathbf{r}_{\mathbf{i}}\right)$ is the radial distribution function. The mean-field approximation for the system with pair interaction takes $g\left(\mathbf{r}, \mathbf{r}_{\mathbf{i}}\right)=1$ and gives a linear dependence of the energy per ion with the global density, $u(\rho)=\Phi_{o} \rho / 2 ; \Phi_{o}$ is the total integral of the interaction potential. The exact result may be written in an intuitive way as $u_{i}=u_{m f}\left(\hat{\rho}_{i}\right)$, where $\hat{\rho}_{i}$ is an effective density:

$$
\hat{\rho}_{i}=\frac{1}{\Phi_{o}} \int d \mathbf{r} \phi\left(\left|\mathbf{r}-\mathbf{r}_{\mathbf{i}}\right|\right) \rho(\mathbf{r}),
$$

which describes the local enviroment of each ion. In a metal-atom fluid the energy cannot be described as pair interactions and the mean-field energy per ion is not a linear function of the average density. However, we still may try to find an approximate form for the energy per ion in terms of a local effective density.

As a preliminary step, which checks the form of this energy in a system with mixture of localized and delocalized electronic states, we carried out exact diagonalizations of tight-binding calculations on a finite body centered cubic (bcc) lattice, with a single orbital per site, and calculated the ground state energy. The samples have a partial occupation of the lattice sites by monovalent atoms (site energy $-E$, of order the alkali ionization potential) and the remaining sites are empty (site energy $0)$; the hopping matrix element between all nn sites $(t)$ is taken to be a constant. A spectrum of realizations were examined choosing randomly disordered lattice-site occupations. Ensembles with a total number of 128 to 1024 sites were examined, at an occupation which ranged from nearly empty to nearly full. Values of $E / t$ from 12 to 24 were examined. In all cases the ensembles yield an electronic structure which depends on the specific realization. For given $E / t$, we calculated the total ground state electronic energy shift (from $E / t$ ) per occupied site 
for all realizations in our ensemble. As a function of the average occupation density, this shift has a scatter over the realizations which is substantial for low densities, see fig.4(a). However, if the data is replotted as a function of the average fractional occupation of nn sites to the occupied ones in each realization, the scatter is found to be reduced by as much as a factor of ten, see fig.4(b). The total ground state energy shift per ion for the entire ensemble can then be described by a single curve. The data can be equally well fitted by a single function which only depends on the fraction of occupied sites with $n$ occupied nn $(n=0$ to 8$)$. Thus, the total ground state energy of the system can be well approximated by a description in terms of an energy $u\left(\hat{\rho}_{i}\right)$ per ion, with a local effective density $\hat{\rho}_{i}$ which gives a measure of the coordination number of each ion.

The independent electron tight-binding model, described above, suggests that the energy per ion for random configurations may be described in terms of the nn configurations of each ion. However, that model is, of course, a very poor representation of a metal-atom fluid. For a given environment of an ion, a local effective density, its energy should be reasonably well described, if the valence electrons are delocalized over the local region, by the previously discussed second order pseudopotential calculation with the electronic exchange, correlation, and screening effects taken into account, and assuming that the local environment is replaced by a macroscopic one of the same density. Our proposal, then, is to use the mean-field energy per ion $u(\rho)$, calculated as in the previous sections, to approximate the energy per ion via $u_{i}=u\left(\hat{\rho}_{i}\right)$; the effective density, $\hat{\rho}_{i}$, is to be a simple function of the coordination number. Thus, for a given ion and limiting the number of its nn to eight (as observed experimentally for high densities and low temperatures), we shall take $\hat{\rho}=(n+1) \rho_{0} / 9$ with $\mathrm{n}$ being the number of its nn (between 0 and 8 ); $\rho_{0}$ is taken to be the density of the liquid metal at the melting point, thus assuming that equilibrium density fluctuations are due to a change in coordination rather than to nn distance changes.

For ions without any nn $(n=0)$, this energy calculated as in a metal is suspect due to the long screening length, so it is compared with that of a free atom (i.e. minus the ionization energy) and in a variational spirit the lower value is chosen (thischoice is along the lines noted in our previous work 1122). Although the calculated energy difference is small in this comparison, the atomic state is lower for all the alkalis. For a single nn, the metallic energy is found to be lower than that of the isolated dimer, for all alkalis. This approach thus includes the structurally-based possibility of valence electron localization, to form atoms, or some degree of delocalization. Results for $\mathrm{u}(\mathrm{n})$ calculated in this manner, for the alkalis, are shown in fig. 5, using the Shaw pseudopotential fitted to one bar at the melting point; an example is also given of results arising from use of the Ashcroft pseudopotential fitted to 0.45 packing fraction at the melting point. These data have the same functional dependence, with coordination, as the tight-binding results quoted previously, as can be appreciated by comparing fig. 5 with the previously noted data in fig. 4; in both cases there is a clearly nonlinear dependence of the energy with local density, reflecting the non-additive character of the interactions arising from valence electron delocalization.

\section{B. Structural background}

Having proposed a recipe to calculate the energy of an ion in a given environment and thus the energy of any inhomogeneous statistical configuration of the system, we wish to use it to self-consistently calculate the equilibrium structures of the materials of interest at chosen thermodynamic conditions. The simplest manner in which to implement the calculations we propose is as follows. A theoretical construction which will yield a material expansion through a change in the average coordination (as observed), which simplifies the study of a fluid with equilibrium density fluctuations, and which is known to allow for a phase transition without broken symmetry, the liquid-vapor type, is a model in which particles (atoms and ions) are constrained to partially occupy the sites in a chosen lattice. Thus disorder is an intrinsic feature of such a treatment and need not be inserted in an adhoc manner, such as a Gaussian, or other, distribution of site energies and/or of transfer elements, typical of Anderson model treatments. As we are interested in treating structural, thermodynamic, and electronic properties on a similar footing, a completely adhoc treatment of the disorder would be inimical to our purpose.

Experimental data 15 on the nn distance and average coordination of coexisting liquid cesium indicates that the lattice gas is an adequate treatment of short-range effects. The measurements show a nn distance which changes little from the liquid at the triple point to nearcritical conditions, while expansion takes place though a changing average coordination number. It is clear, however, that long-range effects due to the imposition of a lattice treatment on a fluid problem will tend to underestimate the system entropy and thus, among other effects, lead to an overestimate of the critical temperature. Nevertheless, such an approach seems a suitable first step. It has been noted that near-critical effects in metal-atom fluids appear to be partially due to nonadditive effects in the interactions 19,20 , a feature we include in the model. Improvements to our model will certainly be possible.

In our model, we allow the ions to partially occupy the sites of a bcc lattice, which allows for the correct coordination of the sites when compared to the dense, alkali, liquid metals. The lattice parameter is determined by the condition that, at full occupation, the maximum density be that of the liquid at the melting point, $\rho_{0}$. The energy for each ion is taken to be $u(\hat{\rho})$; that is, the free energy per ion previously calculated, in the continuum meanfield description, for a macroscopic average density $\hat{\rho}$ and 
setting the temperature to zero. The equilibrium properties are then obtained using grand canonical Monte Carlo simulations; we can then proceed to calculate the equilibrium structures, phase coexistence, and other features of interest.

As a test of the dependence of coexistence data on the functional dependence of $u(\hat{\rho})$, we note the following. A lowest-order approximation scheme, to treat the problem of interest to us, could be based on experimental parameters only by assuming that the energy for an ion with no $\mathrm{nn}$ would be the atomic energy while that for one with the full complement of occupied neighbors (eight) would correspond to the cohesive energy $u_{c}$ of the liquid at the melting point. A linear interpolation for the intermediate cases, $n$ occupied neighbors, would give the equivalent of an Ising model, with a pair potential, at nn only, being given by the cohesive energy of the liquid metal at the melting point (relative to separated atoms) divided by four. The coexistence curve and critical parameters in that case are obtainable using well-known methods 18 . Using the results of the Ising model mapped to a bcc lattice gas yields, for example, $k_{B} T_{c}=0.79385\left(E-u_{c}\right) / 2$. The resulting critical temperatures for the alkalis would then be of order twice the experimental ones for all the alkalis and with the observed systematics, as can be verified by using the experimental cohesive energies of the liquid metals at their melting point (table I). The critical densities resulting from this approximation would then be half of $\rho_{0}$. It should be noted that this approach gives critical temperatures which, though high, are an improvement over the even higher mean-field results. However, the coexistence curve would be found to be symmetrical, in contrast to observations, and the critical densities would be too large. The decrease in the critical temperature of this Ising model, when compared to macroscopic mean-field results, is due to clustering induced by the pair interactions. The symmetry of the coexistence curve arises from the symmetry between occupied and empty sites. Such an approximation scheme fails to take into account many-body effects due to valence electron delocalization, which yield the concave-up $u(n)$ vs $n$ curves. The enhanced cohesion of intermediate clusters, implied by such curvature, further enhances clustering. However, the curvature implies that clusters may be more strongly or more weakly bound, relative to nearby local densities, than the above Ising approach. The local tangent to the $\mathrm{u}(\mathrm{n})$ curve is an effective Ising coupling at that local density; for low $\mathrm{n}$ this coupling is stronger than that due to connecting the end points, and for large $\mathrm{n}$ it is weaker. Thus, although the curvature yields more clustering, the critical temperature due to a model with such curvature need not be higher than that of the straight-line Ising model. Also, the curvature breaks the symmetry between occupied and empty sites. Both effects could yield improvements in calculated data, allowing closer approach to observations. We have found that this is indeed the case. We proceeded to discuss the test of our proposed scheme on various alkalis and a spectrum of their prop- erties.

\section{Monte Carlo Simulations}

We will present the results of grand canonical Monte Carlo simulations for this model, applied to the alkalis from cesium through sodium. First, with an energy per ion taken to be $u(\rho)$, a mean-field lattice treatment, we have compared the critical data with that of the previous, continuous, metallic model to explore the influence of the lattice. The results are given in table II (labeled MF-lg) and are found to be quite close to those of the continuous model; this is due to the low critical density resulting from the mean-field approximation, which makes the entropy of the reference system close to the ideal-gas entropy. However, the crucial point is to take the inhomogeneities in the statistical configurations into account. Simulations were then performed using the energy per ion $u(\hat{\rho})$; this function has been previously discussed and is shown in fig.5. We have used a simulation cube with twelve bcc cells on each side (3456 sites) and periodic boundary conditions. Some results have been checked using a larger cube (8192 sites), without appreciable changes in the results. The simulations, carried out at fixed temperature and chemical potential, give the equilibrium density and internal energy of the system. The pressure is obtained by thermodynamic integration. All results which follow are obtained using the Shaw pseudopotential fitted to 1 bar of pressure at the melting point.

\section{Coexistence}

Calculations of the coexistence curves of the alkalis were carried out. Once again we first examine the critical parameters as an approximate test of the results, before continuing to a more detailed comparison with observations. The critical parameters obtained are also given in table II and labeled as MC. The critical temperatures from our model are higher (by about $400 \mathrm{~K}$ ) than those of the real fluids, a result one might expect from the latticegas treatment of the configurational entropy. However, the results in table II show that the correlation effects included in the present model produce very large decreases of the critical temperatures compared with the previously mentioned mean-field approach, and also compared to the Ising approximation. Also, the critical densities and pressures are brought into reasonable agreement with experiments. Moreover, the relative critical parameters of the various alkalis, in our model, are very similar to the relative experimental ones. These results, it should be noted, also exhibit quantitative improvements relative to those, using the Ashcroft pseudopotential, reported in the table of our letter 5 . Finally, they are used to scale the coexistence curves reported below. 
In fig. 6, we show the coexistence curves, in critical reduced units, calculated and observed for cesium, this data should be compared with that shown in fig. 1 of our previous work 5 . Using the present methods, the shape of the coexistence curve is in good agreement with experiments. It recovers the strong liquid-vapor asymmetry. The figure also presents the diameter function $\rho_{d}=\left(\rho_{L}+\rho_{V}\right) / 2 \rho_{C}$. The errors in the simulations, near critical point, prevent us from a detailed analysis of the deviation of this function from a linear law, observed in the experimental result 3 . We have also calculated the coexistence curves for other alkalis. The results are shown in fig. 7; in reduced critical units, they are all very similar. In agreement with experiments, our results for different alkali fluids give similar reduced phase diagrams. The accuracy in our critical density determinations is poor and does not allow for a discrimination of the small differences observed, 3 in the shape of the coexistence curves. In our calculations, we estimate an uncertainty of \pm 10 percent in $\rho_{c}$ and half that value for $T_{c}$; the relative values among the alkalis should be reliable, as they are all estimated in the same manner. These relative values show the experimentally observed systematics, as can be seen from the comparison shown in table II.

There are no qualitative changes between the coexistence curves reported here and those, using the alternate Ashcroft pseudopotential, of our previous work However, quantitatively, for example, the critical temperatures are lowered, approaching observations, on using the present rather than the previous approach. The reduction of the critical temperatures on using the Shaw, rather than Ashcroft, pseudopotential is about $1200 \mathrm{~K}$, leaving our results about $400 \mathrm{~K}$ too high when compared to experiment. We believe the relative effect is due to the reduction of the discrepancy between the calculated and observed cohesive energies of the liquid metal at the melting point (see table I), the general shape of the $u(n)$ curves staying generally invariant, as can be seen by the example shown in fig. 5 .

\section{Structural features}

Gross structural aspects such as a nearly-linear decrease in the average coordination of ions in the expanded, coexisting, liquid result from this calculation, see fig. 8. The mean-field result (dotted line) underestimates the mean coordination number obtained on approaching the critical point, this is an effect of clustering. The analyses of experimental neutron scattering data 15 , along the liquid line at coexistence, yield pair correlation functions which show a mean-field linear behavior on reducing the density from the triple point to a density of about one-half of that value. Such data, at even lower densities, are difficult to analyze, due to the broadness and lack of definition of the first peak in the de- duced pair correlation function, and, so far, there is no strong evidence for clustering from that data. On the other hand, inverse MC calculations21, based on the experimental structure functions themselves, do show evidence of clustering, even though such calculations tend to underestimate clustering effects because of the averaging inherent in the method. Also, dimerization effects have been inveked to analyze magnetic susceptibility measurements22, though such an analysis is more speculative than the results of the inverse MC. If low density structural measurements are possible, we await their results for further comparison with our theoretical predictions.

Other structural features which result from our calculations are exemplified by data such as that given in fig. 9. The figure shows the fraction of occupied lattice sites within clusters of a given size, as a function of that size. A cluster here is defined as a set of occupied sites which have no nn outside the cluster and which are connected by $n$ within the cluster. The figure is calculated for low density cesium vapor, at the critical temperature obtained from the model and at two average density values: 0.023 and $0.10 \rho_{o}$. The smaller density is well below the critical density and the obtained probability of finding a large cluster decays exponentially with the cluster size. The larger density is near the percolation threshold and the calculated probability of a given cluster decays only as a power of the cluster size. Effects due to such percolation will be discussed later in connection with electrical conductivity estimates. The fraction of the total number of occupied sites in clusters of a given size decreases with the cluster size at fixed average system density. The scatter of points results from data due to various realizations of the MC simulations: small clusters have high probability, they are obtained in most of the realizations and thus show little scatter; large clusters are improbable, they are only observed in some realizations, and thus show a substantial scatter. This latter effect is due to the limited size of the simulation box. Note the reduction in the number of atoms, clusters of size one, as the average system density is increased; there is no jump in this number with density variations, for temperatures above the phase coexistence.

\section{Electrical Conductivity}

We have next explored electronic transport properties related to structure. The experimental signature of a M$\mathrm{nM}$ transition in these systems is a decrease of several orders of magnitude in the conductivity of the expanded fluid. In our model, such behavior is driven by the percolation of the ionic cluster structure, which can be interpreted as leading to macroscopic delocalization of valence electrons, rather than by considerations such as those of Mott or Anderson. Such percolation, however, need not be unrelated to the physical basis in the Mott and An- 
derson pictures. From typical configurations of our MC simulations, we have obtained the cluster structure at different temperatures and pressures. The electrical conductivity was then estimated following the Kirchoff's law model proposed by Nield et al21, with a fitting of the experimental conductivity at the maximum density.

The details of the approach we have used are the following. For a given equilibrium configuration of the occupied sites in our simulation box, nn occupied sites are replaced by a bond resistance of fixed value, no other effects are taken into account. An electrical potential difference is then assumed to be applied to two parallel faces of the box, while periodic boundary conditions are applied to the other sets of box faces. The potential difference would cause a current to flow, through the resistor network, using Ohm's law and requiring that the net current into each site must be zero (Kirchoff's law). The equations are then solved for the current as a function of the potential difference and, taking into account the box size, the conductivity is calculated as a function of the assumed bond resistance. Appropriate averages are carried out for a set of realizations of the simulation at each set of conditions. Clearly a finite conductivity is only found if there is at least one bond-percolation cluster traversing the potential difference. These conductivity estimates are intended to probe the effects of the obtained cluster structure on the transport properties. The estimates are based on an imposed macroscopic treatment of each nn bond in the material, a resistor, instead of a direct quantum treatment. At first glance such an approach appears to be a gross simplification, yet there is some reassurance: the bond resistance required to fit the experimental data at high densities is $15000 \Omega$, a result rather similar to the quantum of resistance, without dissipation, per electron, in a single channel $\left(h /\left(2 e^{2}\right)\right)$ : $12906 \Omega$; the appropriate classical resistance should indeed be greater than the quantum of resistance, due to a transmission coefficient effect. Further justification of this approach is our conjecture that the electron mean free path is rather short in the physical cases, at the conditions considered, so that direct transport between sites separated by distances larger that that of nn sites would only yield negligible contributions.

The results in fig. 10 show the conductivity estimated in this manner along the obtained critical isotherm, for Cs, versus the pressure. They are in excellent agreement with the experimental observations 1 , as is shown in the figure. The figure also shows the calculated conductivity versus density at the critical temperature, and that which would arise from a random occupation of the lattice. The difference clearly shows the effects due to energy-driven clustering. The percolation density (at which the conductivity goes to zero) at $T_{c}$ is less than half of that obtained with random occupation. These results are almost unchanged from those reported in fig. 2 of our letter which arose from the Ashcroft pseudopotential. A line delimiting the densities and temperatures for the percolation onset is shown in fig. 6, accompanying our coex- istence calculations for Cs. It is clear that this line does not intersect the coexistence curve at the critical point, as has been speculated. The results for the percolation line are similar in the spectrum of alkali fluids.

\section{Summary}

We have presented a lattice-gas implementation of a model allowing a unified study of the structural, thermodynamic, and electronic properties of metal-atom fluids. The model takes into account the inhomogeneity of statistical configurations of the system. Nonadditive interactions, due to valence electron delocalization, are included. A self-consistent procedure is used to determine the equilibrium structures: a MC simulation which goes beyond mean-field, as is required. Although the model is a very simplified representation of a metal-atom fluid, comparisons of results obtained, for the alkali family and a spectrum of data, show that the model contains the basic ingredients to allow reproduction of the peculiar behavior observed in these systems. These peculiarities include the M-nM and liquid-vapor transitions and the connection between ionic and electronic structures. Our results for the scaled coexistence curves are in good agreement with observations. The calculated critical temperatures are still somewhat high and the critical pressures low, the densities being adequate. It is reasonable to expect that, with a more realistic (non-lattice) description of the fluid entropy, a similar model (though more cumbersome to study) would give a quantitatively good result for all system properties. Our simplified model appears to allow a unified understanding of the peculiar characteristics of the alkali fluids. The model also shows the similarities and differences between these materials and pairwise interacting ones.

\section{CONCLUSION}

This paper reports investigations of theoretical models which attempt to probe the liquid-vapor and M-nM transitions in the alkali fluids from a unified point of view. Mean-field attempts were investigated first, as an extension of previous work. Although, based on statistical mechanics, they are capable of unifying the two transitions, they are lacking a microscopic basis for treating the coexistence of occupation of the valence electron states localized in atoms and those shared by various ions, which eventually result in accounting for metallic properties. Further, such models do not yield semiquantitative agreement with observations of the structure and coexistence curves of alkali fluids.

Our model was then discussed. It includes treatment of the equilibrium density fluctuations driven by interactions, which include nonadditive effects due to, at least, 
partial valence electron delocalization. This model allows a unified, self-consistent, treatment of structural, thermodynamic, and electronic properties. The latticegas implementation of this model via a $\mathrm{MC}$ simulation method was then undertaken. The results of such a treatment do reproduce the spectrum of peculiar features observed in alkali fluids, including the coupling of structural and electronic properties. It should be emphasized that except for the nonadditive effects, due to valence electron delocalization, the model is no different from that applicable to insulating fluids. Thus we no longer consider that the treatment for both types of materials is intrinsically different, except for effects due to electron delocalization. We believe that our model contains the basic ideas required for an adequate treatment of such fluids.

The energy recipe we have used is a strong simplification of nature, treating electrons which are delocalized over various ions as macroscopically delocalized and also neglecting thermal effects. Further, although our calculations with the model treat the short-range structure of the material in a manner we believe to be reasonably adequate, the long-range structure is oversimplified by the imposition of a lattice. Improvements are required for a full quantitative treatment. The main features of such improvements should seek to give a better treatment of entropy effects with no lattice-like effects in the longrange fluid structure. Such an improvement should be treatable in an improved, non-lattice, MC simulation for a fluid. We urge that such a calculation be undertaken. Also, it would be interesting to attempt an extension of the ideas we have presented in order to seek an improved understanding of polyvalent atoms; mercury can clearly be the testing ground of such extensions. Finally, attempts to integrate Anderson and Mott treatments with the work presented here could yield a deeper understanding of metal-atom fluids. We hope our calculations are a useful stepping-stone to allow guidance of experimental efforts and that extensions of this work will allow theoretical extrapolations to materials at experimental conditions other than those achievable with static techniques.

\section{ACKNOWLEDGMENTS}

We thank Jianping Lu for some computations related to the tight-binding calculations. Our work was partially supported by the Dirección General de Investigación Científica y Técnica (Spain) under Grant PB910090 and the NATO Office of Scientific Research via grant SA.5-2-05(CRG.940240). One of us (JPH) is also grateful for financial support to the W. R. Kenan, Jr. Foundation, the Spanish Ministry of Science and Education, and the Instituto Nicolas Cabrera.
${ }^{1}$ F. Hensel in Physics and Chemistry of Electrons and Ions in Condensed Matter, J. V. Acrivos, N. F. Mott, and A. D. Yoffee, eds. (Dordrecht, Reidel, 1984) p. 401; F. Hensel, S. Jungst, F. Noll, and R. Winter in Localization and Insulator-Metal Transitions, D. Adler and H. Fritzche, eds. (New York, Plenum, 1985) p. 109; F. Hensel, H. Uchtmann, Annu. Rev. Phys. Chem. 40, 61 (1989)

${ }^{2}$ G. Hohl, Ph. D. thesis, Marburg 1992 (unpublished)

${ }^{3}$ S. Jüngst, B. Knuth, F. Hensel, Phys. Rev. Lett. 55, 2164 (1985)

${ }^{4}$ R. M. Stratt, Annu. Rev. Phys. Chem. 41, 175 (1990)

${ }^{5}$ P. Tarazona, E. Chacón, J. P. Hernandez, Phys. Rev. Lett. 74, 142 (1995)

${ }^{6}$ From Hamiltonians to Phase Transitions, Springer series in solid-state sciences 70, by J. Hafner (Springer-Verlag, Heidelberg, 1987)

${ }^{7}$ P. Minchin, M. Watanabe, W. H. Young, J. Phys. F: Metal Phys. 7, 563 (1977)

8 J. P. Hernandez, Phys. Rev. Lett. 57, 3183 (1986)

${ }^{9}$ P. A. Lee, T. V. Ramakrishnan, Rev. Mod. Phys. 57, 287 (1985)

10 See: Quantum Statistics of Charged Particle Systems by W. Kraeft, D. Kremp, W. Ebeling, G. Röpke (Plenum, New York., 1986); R. Redmer, G. Röpke, Physica 130A, 523 (1985), Contrib. Plasma Phys. 29, 343 (1989); R. Redmer and W. W. Warren Jr., Phys. Rev. B 48, 14892 (1993); S. Nagle, G. Röpke, R. Redmer, F. Hensel, J. Phys.: Condens. Matter 6, 2137 (1994)

${ }^{11}$ E. Chacón, J. P. Hernandez, P. Tarazona, J. Phys.: Condens. Matter 5, 1753 (1993)

12 J. P. Hernandez, Z. Phys. Chem. 184, 11 (1994)

13 A brief report was presented at the VII International Workshop on the Physics of Non-Ideal Plasmas (Rostock, 1993): P. Tarazona, E. Chacón, J. P. Hernandez, Contrib. Plasma Phys. 33, 370 (1993)

${ }^{14}$ Liquid Metals by M. Shimoji (Academic Press, New York 1977) p. 28; N. W. Ashcroft and D. Stroud in Sol. St. Phys. 33, H. Ehrenreich, F. Seitz and D. Turnbull, eds. (Academic, New York 1978) p. 1; and ref. 6

${ }^{15}$ R. Winter, C. Pilgrim, F. Hensel, C. Morkel, and W. Glaser, J. Non-Cryst. Solids 156-158, 9 (1993)

${ }^{16}$ J. P. Hernandez, E. Chacón, G. Navascues, Phys. Rev. B 47, 6911 (1993), J. Non-Crystalline Sol. 156-158, 455 (1993)

17 G. A. Mansoori, N. F. Carnahan, K. E. Starling, and T. W. Leland Jr., J. Chem. Phys. 54, 1523 (1971)

${ }^{18}$ M. E. Fisher, Repts. of Prog. in Phys. XXX, 615 (1967)

${ }^{19}$ R. E. Goldstein, N. W. Ashcroft, Phys. Rev. Lett. 55, 2164 (1985)

${ }^{20}$ R. E. Goldstein, A. Parola, A. P. Smith, J. Chem. Phys. 91, 1853 (1989)

${ }^{21}$ V. M. Nield, M. A. Howe, R. L. McGreevy, J. Phys.: Condens. Matter 3, 7519 (1991)

${ }^{22}$ W. Freyland, Phys. Rev. B 20, 5104 (1979) 
FIG. 1. Difference between the mean-field free energy of $\mathrm{Na}$ (Ashcroft pseudopotential) with a HS reference system and that with the OCP (eV) vs density (a.u.), for various temperatures: solid line, $T=2000 K$; long-dashed line, $T=4000 K$; short-dashed line, $T=6000 K$.

FIG. 2. Coexistence curve obtained for metallic rubidium in the mean-field approximation, with a HS reference system. Solid line: Shaw pseudopotential, dotted line: Ashcroft pseudopotential. Temperature in K, density in a.u..

FIG. 3. Coexistence curves obtained for metallic cesium in equilibrium with its atoms, mean-field treatment: The dashed line shows the results on treating the atoms as hard spheres, with a diameter (10a.u.) different than that of the ions (8.80a.u.), but not otherwise interacting with the charges; for this case the temperature scale should be doubled. The full line present the results when, in addition, an empirical interaction with the charges $\left(a \rho_{a} \rho_{i}^{\beta}\right)$ is included. The parameters, $a=-1900 a . u$. and $\beta=1 / 3$, are tuned to minimize the critical temperature. The open points show the experimental coexistence curve of cesium (ref.[3]).

FIG. 4. Ground state, tight-binding, energy shifts per ion $(\Delta E / t)$ calculated for a set of random occupation realizations in a bcc lattice, with $E / t=24$ : (a) as function of the average occupation density, (b) as a function of the average occupation of a nn site for each occupied site in the various realizations.

FIG. 5. Energy per ion in the alkalis, with the Shaw pseudopotential, $u(\rho)$ vs density normalized to density of the liquid metal at the melting point $\rho_{0}$. Solid line: cesium $\left(\rho_{0}=1.84 \mathrm{~g} / \mathrm{cm}^{3}\right)$, dotted line: rubidium $\left(\rho_{0}=1.47 \mathrm{~g} / \mathrm{cm}^{3}\right)$, dashed line: potassium $\left(\rho_{0}=0.828 \mathrm{~g} / \mathrm{cm}^{3}\right)$, and dashed-dotted line: sodium $\left(\rho_{0}=0.924 \mathrm{~g} / \mathrm{cm}^{3}\right)$. The crosses at the end each curve are the experimental cohesive energies. For comparison, the circles show the results for Cs using the Ashcroft pseudopotential, which were used in ref. [5]. Energies are in eV. The $\rho / \rho_{0}=1 / 9$ (i.e., $\mathrm{n}=0$ ) values are experimental data for the free atoms.

FIG. 6. Liquid-vapor coexistence curve of cesium, in reduced critical units. Filled circles: present MC simulation; full line: fit to the experimental results of ref.[3]. The MC simulation diameter function $\rho_{d}$ is also plotted (crosses). The triangles, joined by a line to guide the eye, delimit the region of cluster percolation. Note the slight differences with fig. 1 of ref. [5], due to a change in the pseudopotential choice.

FIG. 7. Calculated liquid-vapor coexistence curves for the alkali fluids, using the Shaw parametrization, in reduced critical units; circles: cesium, squares: rubidium, triangles: potassium, and stars: sodium.
FIG. 8. Average coordination number versus normalized density, along the the liquid branch of the coexistence curve of cesium, resulting from the MC treatment of the lattice gas. The dotted line shows the mean-field result, with average coordination number equal to $8 \rho / \rho_{0}$.

FIG. 9. Fraction of ions in clusters of each cluster size (number of ions) in a log-log plot. The calculations use the MC lattice-gas treatment, for cesium at the critical temperature, at two average densities: $0.023 \rho_{o}$ (solid points) and $0.10 \rho_{o}$ (open points).

FIG. 10. On the left we show the electrical conductivity $\sigma$ for cesium versus the reduced pressure along the critical isotherm. Crosses from our model, open circles are experimental values from ref [1], both normalized to the conductivity $\sigma_{0}$ at our highest density $\rho_{0}$. On the right we show our calculated electrical conductivity but now versus the normalized density; points are our results for $T=T_{C}$, and triangles for the random occupation of the lattice. Note the slight differences with fig. 2 of ref. [5], due to a change in the pseudopotential choice. 
TABLE I. Comparison of the calculated results for the liquid alkali metals, at their melting point, using the Ashcroft and Shaw pseudopotentials (the core radii marked by an asterisk are fitted to a 0.45 packing fraction for the liquid metal at the melting point). Core radius $\left(r_{c}\right)$ and hard-sphere diameter $\left(d_{H S}\right)$ in a.u., packing fraction $(\eta)$ and excess entropy $\left(S_{E} / k_{B}\right.$ ) are dimensionless, atomic energy $(E)$ and liquid cohesive energy $(u)$ (referred to ion cores and valence electrons at infinite separation) in $\mathrm{eV}$, conductivity $(\sigma)$ in $10^{5}(\Omega \mathrm{cm})^{-1}$, and pressure $(P)$ in bar. Note the systematic improvement in $u$, and to a lesser extent in $E$, on fitting to $P$ - Shaw rather than to $\sigma$ (or packing fraction) - Ashcroft.

\begin{tabular}{|c|c|c|c|c|c|c|c|c|c|c|c|c|c|c|c|c|}
\hline & \multicolumn{8}{|c|}{$\overline{\text { CESIUM }}$} & \multicolumn{8}{|c|}{ RUBIDIUM } \\
\hline & $r_{c}$ & $d_{H S}$ & $\eta$ & $E$ & $u$ & $\sigma$ & $S_{E} / k_{B}$ & $P$ & $r_{c}$ & $d_{H S}$ & $\eta$ & $E$ & $u$ & $\sigma$ & $S_{E} / k_{B}$ & $P$ \\
\hline Ashcroft & $2.62^{*}$ & 8.80 & 0.45 & -3.89 & -5.12 & 0.70 & 3.81 & -6260 & $2.40^{*}$ & 8.19 & 0.45 & -4.23 & -5.39 & 0.53 & 3.81 & -7380 \\
\hline Shaw & 4.73 & 8.82 & 0.44 & -3.93 & -4.73 & 0.68 & 3.79 & 1.0 & 4.30 & 8.18 & 0.44 & -4.16 & -5.05 & 0.64 & 3.77 & 1.0 \\
\hline \multirow[t]{2}{*}{$\operatorname{Exp}$} & - & - & - & -3.89 & -4.69 & 0.28 & 3.56 & 1.0 & - & - & - & -4.18 & -5.01 & 0.45 & 3.63 & 1.0 \\
\hline & $r_{c}$ & $d_{H S}$ & $\eta$ & $E$ & $u$ & $\sigma$ & $S_{E} / k_{B}$ & $P$ & $r_{c}$ & $d_{H S}$ & $\eta$ & $E$ & $u$ & $\sigma$ & $S_{E} / k_{B}$ & $P$ \\
\hline Ashcroft & 2.12 & 7.51 & 0.42 & -4.48 & -5.76 & 0.60 & 3.40 & -9230 & 1.70 & 6.17 & 0.44 & -4.86 & -6.61 & 1.0 & 3.80 & -10700 \\
\hline Shaw & 3.92 & 7.60 & 0.43 & -4.37 & -5.36 & 0.63 & 3.65 & 1.0 & 2.92 & 6.11 & 0.43 & -4.97 & -6.45 & 0.63 & 3.50 & 1.0 \\
\hline $\operatorname{Exp}$ & - & - & - & -4.34 & -5.26 & 0.77 & 3.45 & 1.0 & - & - & - & -5.14 & -6.25 & 1.0 & 3.45 & 1.0 \\
\hline
\end{tabular}

TABLE II. Estimated critical conditions: using continuum mean-field with hard-sphere (MF-hs) and lattice-gas (MF-lg) reference system entropy, Monte Carlo (MC) simulation for the present theory (Shaw pseudopotential), and the experimental results of ref. [2] and [3]. The temperature $T$ is in Kelvin, the pressure $P$ in bar, and the density $\rho$ in $\mathrm{g} \mathrm{cm}^{-3}$. The MC results are later used to normalize the results presented in various figures.

\begin{tabular}{|c|c|c|c|c|c|c|}
\hline & \multicolumn{3}{|c|}{$\overline{\text { CESIUM }}$} & \multicolumn{3}{|c|}{ RUBIDIUM } \\
\hline & $T_{c}$ & $\rho_{c}$ & $P_{c}$ & $T_{c}$ & $\rho_{c}$ & $P_{c}$ \\
\hline MF-hs & 6330 & 0.022 & 6.7 & 6820 & 0.019 & 9.3 \\
\hline MF-lg & 6540 & 0.030 & 8.3 & 7060 & 0.025 & 11.9 \\
\hline $\mathrm{MC}$ & 2350 & 0.47 & 60 & 2475 & 0.35 & 73 \\
\hline \multirow[t]{3}{*}{ Exp } & 1924 & 0.38 & 92.5 & 2017 & 0.29 & 124.5 \\
\hline & \multicolumn{3}{|c|}{$\overline{\text { POTASSIUM }}$} & \multicolumn{3}{|c|}{$\overline{\overline{\text { SODIUM }}}$} \\
\hline & $T_{c}$ & $\rho_{c}$ & $P_{c}$ & $T_{c}$ & $\rho_{c}$ & $P_{c}$ \\
\hline MF-hs & 7310 & 0.011 & 13.0 & 9020 & 0.014 & 34.6 \\
\hline MF-lg & 7580 & 0.014 & 16.2 & 9390 & 0.018 & 45.4 \\
\hline $\mathrm{MC}$ & 2550 & 0.22 & 70 & 2970 & 0.22 & 128 \\
\hline $\operatorname{Exp}$ & 2178 & 0.18 & 148 & 2485 & 0.30 & 248 \\
\hline
\end{tabular}

